\title{
Boundary element approaches for acoustic computation of perforated silencers
}

\author{
Liang Yang \& Zhenlin Ji \\ College of Power and Energy Engineering, Harbin Engineering \\ University, P. R. China
}

\begin{abstract}
Substructure boundary element method, direct mixed-body boundary element method, and substructure direct mixed-body BEM are used to calculate the acoustic attenuation characteristics of silencers with perforated facing. The transmission loss predictions of straight-through perforated tube silencers by the three approaches agree well with experimental measurements in the literature. The computational efficiency is compared in the paper which shows that the substructure direct mixed-body BEM can save a large amount of computation time for the large muffler contains complex internal geometry. Test case of a hybrid expansion chamber silencer with perforated facing is analyzed in the paper.

Keywords: boundary element method, substructure approach, direct mixedbody BEM, perforated silencers, computational efficiency.
\end{abstract}

\section{Introduction}

Mufflers and silencers with perforated facing are widely used to attenuate the intake and exhaust noise power machineries. Three-dimensional numerical technique such as finite element method (FEM) $[1,2]$ and boundary element method (BEM) [3-7] are suitable to predict the acoustic performance of mufflers due to the advantage of no limitations for geometry. The BEM is an ideal analysis tool for silencer design because of its surface-only meshing scheme. For reactive silencers with extended inlet/outlet tubes, thin baffles and perforated tubes or dissipative silencers with sound-absorbing materials, substructure BEM approach together with the impedance matrix synthesis was used by $\mathrm{Ji}$ et al. $[3,7]$ to calculate the transmission loss. Wu and his co-workers $[4,5]$ 
developed the so-called direct mixed-body BEM to overcome the drawback of tedious zoning matching steps of substructure BEM. Lou et al. [6] also combined the direct mixed-body with impedance matrix synthesis to reduce the matrix size of large geometry problems. A packed simple expansion chamber silencer was used to demonstrate the substructuring technique and the computational efficiency was tested. With the direct mixed-body BEM, each substructure may contain complex components and does not need to be homogeneous. The direct mixed-body BEM ignores the thickness of thin-wall and perforated components while the substructure BEM can consider this.

In the present paper, the substructure BEM, the direct mixed-body BEM and the substructure direct mixed-body BEM with impedance matrix method are used to calculate the transmission loss of silencers with perforated facing. Calculated TL results of straight-through perforated tube silencers by the three approaches are compared with experimental measurements. Computational efficiency of the three approaches is examined for a hybrid expansion chamber silencer with perforated facing.

\section{Boundary element method}

The boundary integral equation can be written as

$$
C\left(r_{p}\right) p\left(r_{p}\right)=\int_{\Gamma}\left(G\left(r_{p}, r_{Q}\right) \frac{\partial p\left(r_{Q}\right)}{\partial n_{Q}}-\frac{\partial G\left(r_{p}, r_{Q}\right)}{\partial n_{Q}} p\left(r_{Q}\right)\right) d S,
$$

where $\partial / \partial n_{Q}$ denotes the normal derivative, and $G$ is the Green's function given by

$$
G\left(r_{p}, r_{Q}\right)=\exp \left(j k r_{p Q}\right) / 4 \pi r_{p Q} .
$$

After meshing the surface and considering the boundary conditions, Eqn (1). can be expressed as the following linear matrix form

$$
[H+I]\{P\}=[G]\{U\}
$$

where $[H],[I]$ and $[G]$ are the coefficient matrices with dimension of $N \times N, N$ is the number of discretizing nodes on the boundary, and $\{P\}$ and $\{U\}$ are the vectors of acoustic pressure and normal particle velocity on the boundary nodes, respectively. The coefficients in the matrices can be expressed as

$$
\begin{gathered}
I_{i j}=1 / 2 \delta_{i j}, \\
H_{i j}=\int_{\Gamma} N_{j}\left(r_{Q}\right) \frac{\partial G\left(r_{i}, r_{Q}\right)}{\partial\left(n_{Q}\right)} d S_{Q}, \\
G_{i j}=j k z \int_{\Gamma} N_{j}\left(r_{Q}\right) G\left(r_{i}, r_{Q}\right) d S_{Q},
\end{gathered}
$$


where $\delta$ is Kronecker's delta function, $r_{i}$ is the position vector of node $i, N_{j}$ is the interpolation function of node $j, k$ is the wavenumber and $z$ is the characteristic impedance of the medium, respectively.

\subsection{Substructure boundary element method}

The traditional boundary element method (CBEM) is suitable for the single domain and homogeneous acoustic problems. In practical design, silencers always contain the so-called degenerate boundary such as extended inlet/outlet tubes, thin baffles and perforated tubes. Combining the CBEM with substructure technique, the substructure boundary element method can be built. It can deal with the degenerate boundary difficulty and is suitable for dissipative silencers with sound-absorbing material. In addition, for the large-scale acoustic problems, the substructure BEM can improve the computational accuracy and efficiency.

According to the structure and media distribution characteristics, the acoustic field may be divided into several homogeneous and well-defined subdomains. The Helmholtz integral equation can be written for each individual subdomain. The continuous condition of sound pressure and particle velocity in the interface is used to assemble the matrices of each substructure. Combined with the boundary conditions, unknown on each node can be calculated.

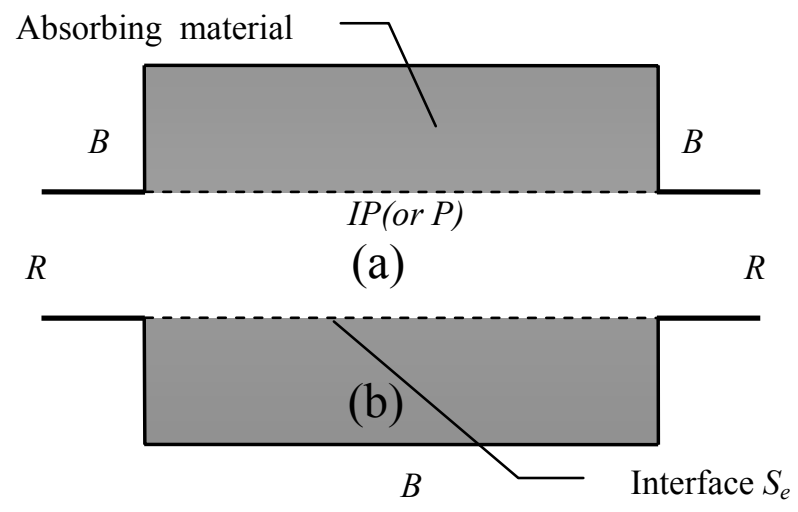

Figure 1: Sound field domain division of straight-through perforated tube silencer.

Taking the straight-through perforated tube dissipative silencer shown in fig. las an example, there are two kinds of media in the silencer: air and soundabsorbing material. Virtual boundary $S_{e}$ (the perforated face) divides the acoustic domain into two subdomains (a) and (b). Each substructure contains real boundaries and virtual boundaries. Applying boundary element method to each substructure and discretizing the boundaries, the following linear equations can be obtained 


$$
\begin{aligned}
& {\left[\begin{array}{ll}
H_{r r}^{a} & H_{r e}^{a} \\
H_{e r}^{a} & H_{e e}^{a}
\end{array}\right]\left\{\begin{array}{c}
P_{r}^{a} \\
P_{e}^{a}
\end{array}\right\}=\left[\begin{array}{ll}
G_{r r}^{a} & G_{r e}^{a} \\
G_{e r}^{a} & G_{e e}^{a}
\end{array}\right]\left\{\begin{array}{c}
V_{r}^{a} \\
V_{e}^{a}
\end{array}\right\},} \\
& {\left[\begin{array}{ll}
H_{r r}^{b} & H_{r e}^{b} \\
H_{e r}^{b} & H_{e e}^{b}
\end{array}\right]\left\{\begin{array}{c}
P_{r}^{b} \\
P_{e}^{b}
\end{array}\right\}=\left[\begin{array}{ll}
G_{r r}^{b} & G_{r e}^{b} \\
G_{e r}^{b} & G_{e e}^{b}
\end{array}\right]\left\{\begin{array}{c}
V_{r}^{b} \\
V_{e}^{b}
\end{array}\right\} .}
\end{aligned}
$$

$H_{x y}^{k}$ and $G_{x y}^{k}$ are influence coefficient matrices between node set $x$ and node set $y$ in substructure $k . P_{x}^{k}$ and $V_{x}^{k}$ are pressure column vector and particle velocity column vector in substructure $k$, respectively. Considering the perforated facing in the interface, the following relationship can be written,

$$
\begin{gathered}
V_{e}^{a}=-V_{e}^{b}, \\
P_{e}^{b}=P_{e}^{a}-z_{0} \zeta_{p} V_{e}^{a},
\end{gathered}
$$

where $z_{0}$ is the characteristic impedance of air and $\zeta_{p}$ is the specific acoustic impedance for the perforated facing. The expression of $\zeta_{p}$ can be found in the literature [7]. Therefore, eqn. (7) and eqn. (8) may be combined and rewritten as

$$
\begin{gathered}
{\left[\begin{array}{cccc}
H_{r r}^{a} & H_{r e}^{a} & -G_{r e}^{a} & 0 \\
H_{e r}^{a} & H_{e e}^{a} & -G_{e e}^{a} & 0 \\
0 & H_{r e}^{b} & G_{r e}^{b}-z_{0} \zeta_{p} H_{r e}^{b} & H_{r r}^{b} \\
0 & H_{e e}^{b} & G_{e e}^{b}-z_{0} \zeta_{p} H_{e e}^{b} & H_{e r}^{b}
\end{array}\right]\left\{\begin{array}{c}
P_{r}^{a} \\
P_{e}^{a} \\
V_{e}^{a} \\
P_{r}^{b}
\end{array}\right\}=} \\
{\left[\begin{array}{cccc}
G_{r r}^{a} & 0 & 0 & 0 \\
G_{e r}^{a} & 0 & 0 & 0 \\
0 & 0 & 0 & G_{r r}^{b} \\
0 & 0 & 0 & G_{e r}^{b}
\end{array}\right]\left\{\begin{array}{c}
V_{r}^{a} \\
0 \\
0 \\
V_{r}^{b}
\end{array}\right\} .}
\end{gathered}
$$

The coefficient matrices are banded, thus the characteristics of matrices may be improved to some extent.

\subsection{Direct mixed-body boundary element method}

The basic idea of the direct mixed-body BEM actually comes from the conventional multi-domain BEM by summing up the homogeneous and welldefined subdomains to create a single integral equation. The hypersingular integral equation is used to provide an additional equation at the surfaces which have two unknown variables.

For the straight-through perforated tube silencer as shown in fig. 1, there are four kinds of surface: regular $(R)$ surfaces include the exterior surfaces, the inlet/outlet tubes and the inlet/outlet ends, bulk $(B)$ surfaces are the exterior surfaces with the sound-absorbing material, perforated $(P)$ surfaces represent 
perforated surfaces with air on both sides, perforated interfaces (IP) are designated for the perforated interfaces between the sound-absorbing material and air. The complete boundary integral equations for the silencer with perforated facing can be expressed as [5]

$$
\begin{aligned}
& \int_{R}\left(p\left(r_{Q}\right) \frac{\partial G_{A}\left(r_{p}, r_{Q}\right)}{\partial n_{Q}}+j k_{A} z_{A} v_{n}\left(r_{Q}\right) G_{A}\left(r_{p}, r_{Q}\right)\right) d S \\
& +\int_{P} \frac{\partial G_{A}\left(r_{p}, r_{Q}\right)}{\partial n_{Q}}\left(p^{+}-p^{-}\right) d S \\
& +\int_{B}\left(p\left(r_{Q}\right) \frac{\partial G_{B}\left(r_{p}, r_{Q}\right)}{\partial n_{Q}}+j k_{B} z_{B} v_{n}\left(r_{Q}\right) G_{B}\left(r_{p}, r_{Q}\right)\right) d S \\
& +\int_{I P}\left[\begin{array}{c}
p_{A}\left(\frac{\partial G_{A}\left(r_{p}, r_{Q}\right)}{\partial n_{Q}}-\frac{\partial G_{B}\left(r_{p}, r_{Q}\right)}{\partial n_{Q}}\right)-z_{A} \zeta_{p} v_{n}\left(r_{Q}\right) \frac{\partial G_{B}\left(r_{p}, r_{Q}\right)}{\partial n_{Q}} \\
\left.+j k_{A} z_{A} v_{n}\left(r_{Q}\right) G_{A}\left(r_{p}, r_{Q}\right)-j k_{B} z_{B} v_{n}\left(r_{Q}\right) G_{B}\left(r_{p}, r_{Q}\right)\right] d S \\
p\left(r_{p}\right), p \in \Omega \\
0.5 p\left(r_{p}\right), p \in R+B \\
0.5\left[p^{+}\left(r_{p}\right)+p^{-}\left(r_{p}\right)\right], p \in P \\
p r_{A}\left(r_{p}\right)+0.5 z_{A} k_{A} \zeta_{p} v_{n}\left(r_{p}\right), p \in I P
\end{array}\right.
\end{aligned}
$$

and

$$
\begin{aligned}
& \int_{R}\left(p\left(r_{Q}\right) \frac{\partial^{2} G_{A}\left(r_{p}, r_{Q}\right)}{\partial n_{Q} \partial n_{p}}+j k_{A} z_{A} v_{n}\left(r_{Q}\right) \frac{\partial^{2} G_{A}\left(r_{p}, r_{Q}\right)}{\partial n_{p}}\right) d S \\
& +\int_{P} \frac{\partial^{2} G_{A}\left(r_{p}, r_{Q}\right)}{\partial n_{Q} \partial n_{p}}\left(p^{+}-p^{-}\right) d S \\
& +\int_{B}\left(p\left(r_{Q}\right) \frac{\partial^{2} G_{B}\left(r_{p}, r_{Q}\right)}{\partial n_{Q} \partial n_{p}}+j k_{B} z_{B} v_{n}\left(r_{Q}\right) \frac{\partial G_{B}\left(r_{p}, r_{Q}\right)}{\partial n_{p}}\right) d S \\
& +\int_{I P}\left[p_{A}\left(\frac{\partial^{2} G_{A}\left(r_{p}, r_{Q}\right)}{\partial n_{Q} \partial n_{p}}-\frac{\partial^{2} G_{B}\left(r_{p}, r_{Q}\right)}{\partial n_{Q} \partial n_{p}}\right)-z_{A} \zeta_{p} v_{n}\left(r_{Q}\right) \frac{\partial^{2} G_{B}\left(r_{p}, r_{Q}\right)}{\partial n_{Q} \partial n_{p}}\right. \\
& \left.+j k_{A} z_{A} v_{n}\left(r_{Q}\right) \frac{\partial G_{A}\left(r_{p}, r_{Q}\right)}{\partial n_{p}}-j k_{B} z_{B} v_{n}\left(r_{Q}\right) \frac{\partial G_{B}\left(r_{p}, r_{Q}\right)}{\partial n_{p}}\right] d S
\end{aligned}
$$




$$
=\left\{\begin{array}{c}
\frac{j k_{A}}{\zeta_{p}}\left[p^{+}\left(r_{p}\right)-p^{-}\left(r_{p}\right)\right], p \in P . \\
-0.5\left(j k_{A} z_{A}+j k_{B} z_{B}\right) v_{n}\left(r_{p}\right), p \in I P,
\end{array}\right.
$$

where $G_{A}$ and $G_{B}$ are the free-space Green's functions in the air and the soundabsorbing material, respectively. The explicit expressions for the two functions are

$$
\begin{aligned}
& G_{A}\left(r_{p}, r_{Q}\right)=\exp \left(j k_{A} r_{p Q}\right) / 4 \pi r_{p Q}, \\
& G_{B}\left(r_{p}, r_{Q}\right)=\exp \left(j k_{B} r_{p Q}\right) / 4 \pi r_{p Q},
\end{aligned}
$$

$k_{A}$ and $k_{B}$ are the wavenumbers of air and sound-absorbing material, respectively, $z_{A}$ and $z_{B}$ are the characteristic impedances of air and soundabsorbing material, respectively. Eqn. (12) and Eqn. (13) together form the complete set of integral equations necessary to calculate the acoustic characteristics of silencers with perforated facing.

\subsection{Impedance matrix technique}

To begin with, a simple structure is considered which is divided into two structures as shown in fig. 2.

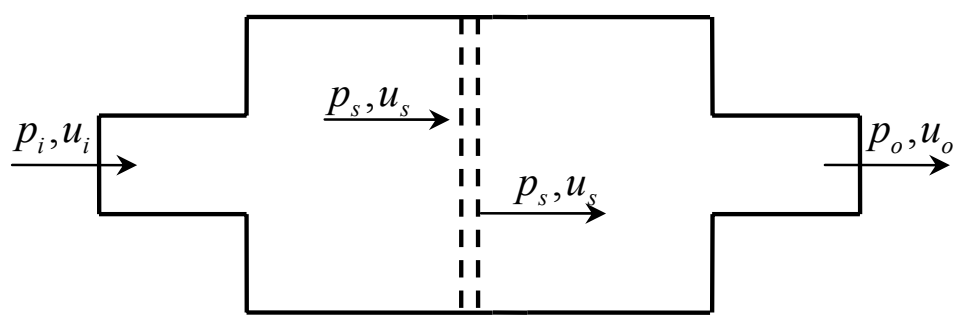

substructure 1

substructure 2

Figure 2: $\quad$ Two substructures with shared boundary.

The sound pressures at the inlet and the outlet of structure 1 are related to the corresponding particle velocities by an impedance matrix

$$
\left[\begin{array}{l}
p_{i} \\
p_{s}
\end{array}\right]=\left[\begin{array}{ll}
Z_{11}^{1} & Z_{12}^{1} \\
Z_{21}^{1} & Z_{22}^{1}
\end{array}\right]\left[\begin{array}{l}
v_{i} \\
v_{s}
\end{array}\right] .
$$

To obtain the first column of the impedance matrix, $v=1$ is applied to the first element at the inlet of substructure 1 , and $v=0$ is applied elsewhere. The sound pressure at the inlet and outlet will become the first column of the impedance matrix. Similarly, $v=1$ is made on each element at the inlet and outlet one at a time, the whole impedance can be obtained.

Similarly, the impedance matrix for substructure 2 can be created. That is, 


$$
\left[\begin{array}{l}
p_{s} \\
p_{o}
\end{array}\right]=\left[\begin{array}{ll}
Z_{11}^{2} & Z_{12}^{2} \\
Z_{21}^{2} & Z_{22}^{2}
\end{array}\right]\left[\begin{array}{l}
v_{s} \\
v_{o}
\end{array}\right],
$$

$p_{s}$ and $v_{s}$ in Eqn. (15) and Eqn. (16) are vector matrices of sound pressures and particle velocities on the shared boundary.

The impedance matrix between inlet and outlet of the whole structure is

$$
\left[\begin{array}{l}
p_{i} \\
p_{o}
\end{array}\right]=\left[\begin{array}{ll}
Z_{11} & Z_{12} \\
Z_{21} & Z_{22}
\end{array}\right]\left[\begin{array}{c}
v_{i} \\
v_{o}
\end{array}\right],
$$

where

$$
\begin{aligned}
& Z_{11}=Z_{11}^{1}-Z_{12}^{1}\left(Z_{11}^{2}+Z_{22}^{1}\right)^{-1} Z_{21}^{1} \\
& Z_{12}=Z_{12}^{1}\left(Z_{11}^{2}+Z_{22}^{1}\right)^{-1} Z_{12}^{2} \\
& Z_{21}=Z_{21}^{2}\left(Z_{11}^{2}+Z_{22}^{1}\right)^{-1} Z_{21}^{1} \\
& Z_{22}=Z_{22}^{2}-Z_{21}^{2}\left(Z_{11}^{2}+Z_{22}^{1}\right)^{-1} Z_{12}^{2} .
\end{aligned}
$$

\section{Results and discussions}

The substructure BEM, the direct mixed-body BEM and the direct mixed-body BEM with impedance matrix method are used to calculate the transmission loss of a straight-through perforated tube reactive silencer (as fig. 3 shows). For the reactive silencer, the diameter $(d)$ of the inlet and the outlet tubes is $0.049 \mathrm{~m}$, the length $(l)$ and the diameter $(D)$ of the chamber are $0.2572 \mathrm{~m}$ and $0.1644 \mathrm{~m}$, respectively. The wall thickness of perforated tube is $0.0009 \mathrm{~m}$ and the diameter of hole is $0.00498 \mathrm{~m}$. The porosity $(\phi)$ is $8.4 \%$.

The transmission loss predictions from the three approaches are plotted in fig.4. It may be seen that all predictions agree well with the experimental data. It seems that the substructure technique can improve the computational accuracy of the direct mixed-body BEM to some extent.

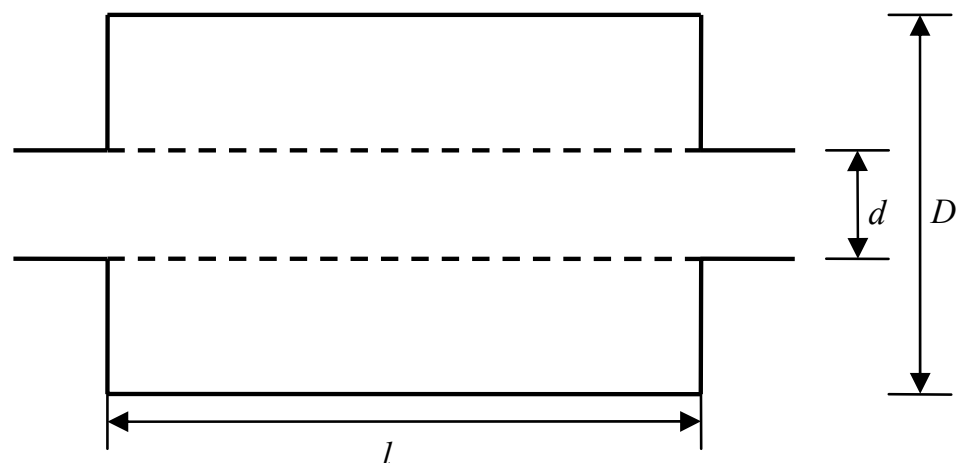

Figure 3: Straight-through perforated tube reactive silencer. 


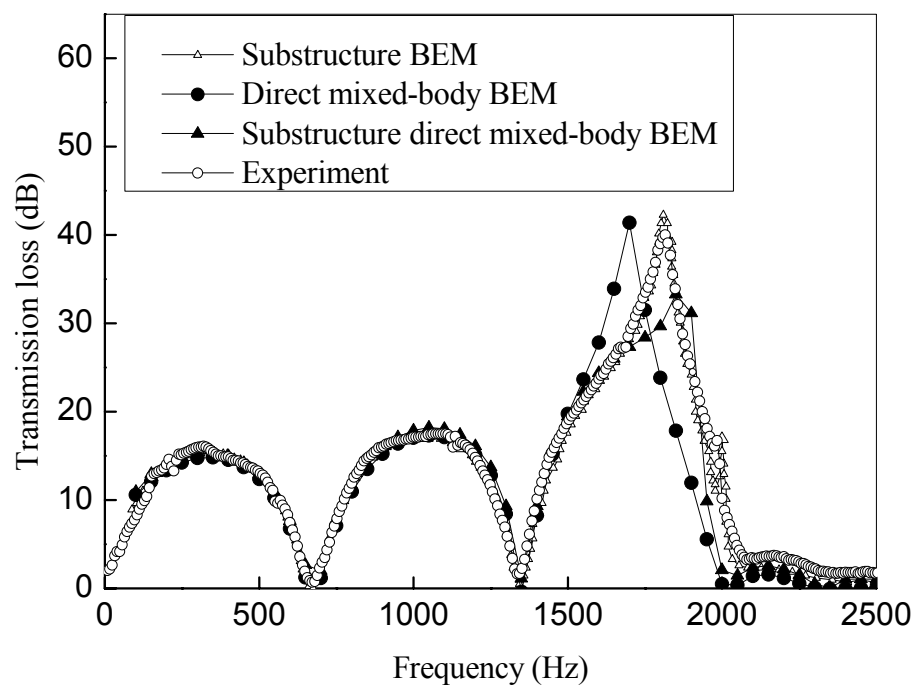

Figure 4: Transmission loss of straight-through perforated tube reactive silencer $(\phi=8.4 \%)$.

In order to show the computational efficiency of the three approaches, table 1 compares the CPU time for the straight-through perforated tube reactive silencer at frequency $f=1 \mathrm{kHz}$. The comparison shown in table 1 was done on a $\mathrm{PC}$ with Pentium dual E2220 2.4 GHz. Note that the computational efficiency of the substructure direct mixed-body BEM is related to the dimension of matrices in Eqn. (15) and Eqn. (16). The more elements picked up in the inlet and the outlet of each substructure, the longer it will take to calculate the transmission loss.

Table 1: CPU time comparison for straight-through perforated tube reactive silencer at $1 \mathrm{kHz}$.

\begin{tabular}{lcc}
\hline Calculation method & Number of elements & CPU time (s) \\
\hline Substructure BEM & $648+2260$ & 56.86 \\
Direct mixed-body BEM & 2140 & 25.00 \\
Substructure direct mixed-body & $1000+1000$ & 18.76 \\
BEM (with two substructures) & & \\
\hline
\end{tabular}

The dissipative silencer with the same dimension as fig. 3, except the soundabsorbing material is filled in the chamber, is considered as the second example. The texturized fiberglass roving is used as sound-absorbing material in the study. 
The complex acoustic impedance $z_{B}$ and the complex wavenumber $k_{B}$ of the material with the filling density of $100 \mathrm{~kg} / \mathrm{m}^{3}$ are expressed as [8]

$$
\begin{aligned}
& z_{B} / z_{A}=1.0+33.20 f^{-0.7523}-j 28.23 f^{-0.6512}, \\
& k_{B} / k_{A}=1.0+39.20 f^{-0.6841}-j 38.39 f^{-0.6285} .
\end{aligned}
$$

Fig. 5 shows the transmission loss predictions of the dissipative silencer. Both the substructure BEM and the direct mixed-body BEM produce decent accuracy.

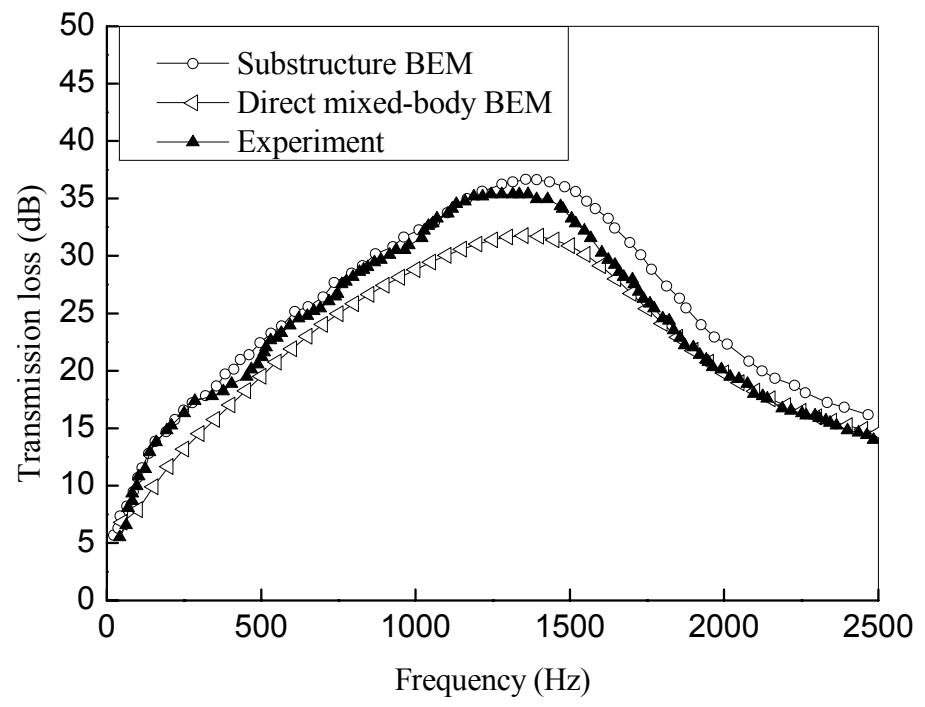

Figure 5: Transmission loss of straight-through perforated tube dissipative silencer $(\phi=8.4 \%)$.

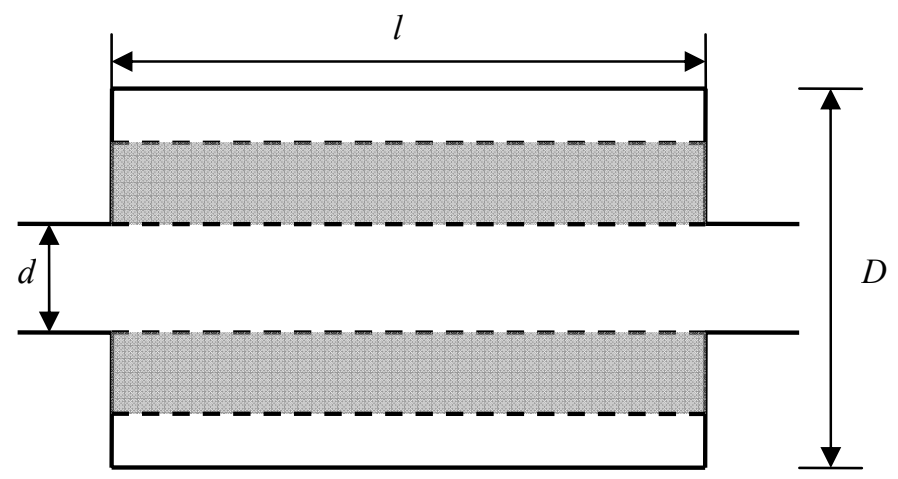

Figure 6: Hybrid expansion chamber silencer. 
The third test case is a hybrid expansion chamber silencer as shown in fig. 6 . The comparison of numerical predictions and measurement is shown in fig. 7. Again, the BEM predictions are in fairly good agreement with the measured TL results.

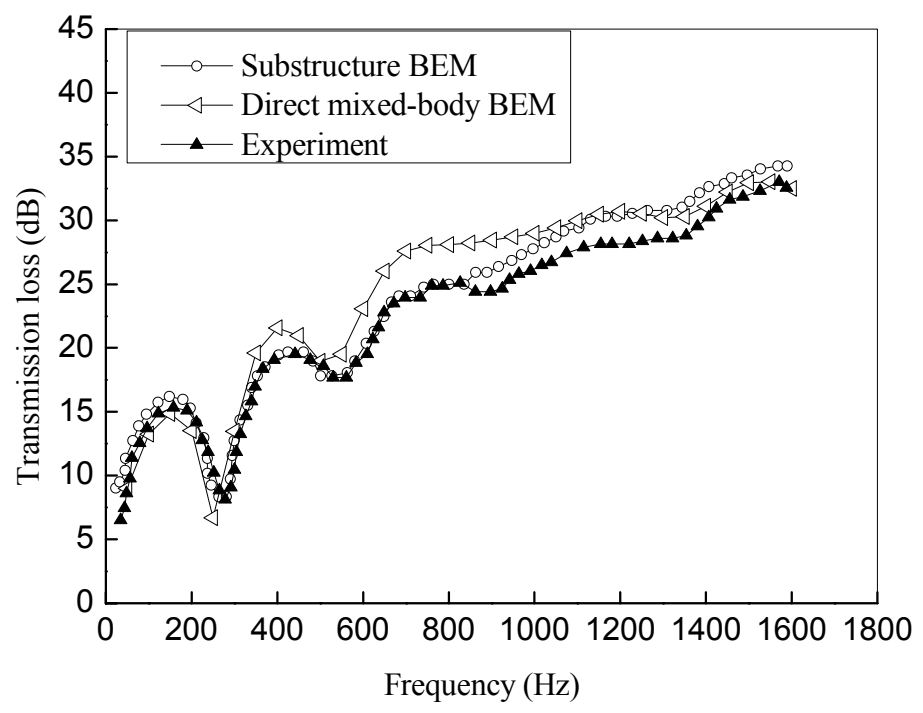

Figure 7: Transmission loss of hybrid expansion chamber silencer $(\phi=8.4 \%)$.

\section{Conclusions}

The substructure BEM, the direct mixed-body BEM and the substructure direct mixed-body BEM are introduced to predict the transmission loss of silencers with perforated facing. The direct mixed-body BEM eliminates the tedious interface matching steps in the substructure BEM. Combining the direct mixedbody BEM with the impedance matrix technique can improve the computational efficiency of perforated silencers.

\section{References}

[1] Peat KS, Rathi KL. A finite element analysis of the convected acoustic wave motion in dissipative silencers. J Sound Vib, 184, pp. 529-45, 1995.

[2] Fang Z, Ji ZL. Finite element analysis of transversal modes and acoustic attenuation characteristics of perforated tube silencers. Noise Control Eng J, 60, pp. 340-49, 2012. 
[3] Ji ZL, Ma Q, Zhang ZH. Application of the boundary element method to predicting acoustic performance of expansion chamber mufflers with mean flow. J Sound Vib, 173, pp. 57-71, 1994.

[4] Wu TW, Zhang P, Cheng CYR. Boundary element analysis of mufflers with an improved method for deriving the four-pole parameters. J Sound Vib, 217, pp. 767-79, 1998.

[5] Wu TW, Cheng CYR, Zhang P. A direct mixed-body boundary element method for packed silencers. J Acoust Soc Am, 111, pp. 2566-72, 2002.

[6] Lou G, Wu TW, Cheng CYR. Boundary element analysis of packed silencers with a substructuring technique. Eng Anal Boundary Elem, 27, pp. 643-53, 2003.

[7] Ji ZL. Boundary element acoustic analysis of hybrid expansion chamber silencers with perforated facing. Eng Anal Boundary Elem, 34, pp. 690-96, 2010.

[8] Lee IJ, Selamet A, Huff NT. Acoustic impedance of perforations in contact with fibrous material. J Acoust Soc Am, 119, pp. 2785-97, 2006. 